\title{
Erratum to: The Spectral Type of the Ionizing Stars and the Infrared Fluxes of HII Regions
}

\author{
A. P. Topchieva*1, M. S. Kirsanova**1,2, and A. M. Sobolev ${ }^{* * * 2}$ \\ ${ }^{1}$ Institute of Astronomy, Russian Academy of Sciences, Moscow, 119017 Russia \\ ${ }^{2}$ K.A. Barkhatova Kourovka Astronomical Observatory, B.N. Yeltsin Ural Federal University, \\ Ekaterinburg, 620000 Russia \\ Received February 15, 2019; revised February 27, 2019; accepted February 28, 2019
}

DOI: $10.1134 / \mathrm{S} 1063772919070023$

Several errors were found in this paper, which however did not influence its main conclusions. In Table 2 of Section 2, the references for the data in columns (6)-(9) and (10)-(11) were given incorrectly, and incorrect values for $T_{\text {eff }}$ were given in column (10). These errors were discovered by the authors in the course of using the data in other studies, and do not make a signficant contributions to the figures, results, or conclusions of this paper. A corrected version of this table with correct references is given below.

Table 2. Distances, sizes, and electron densities of the objects studied. The ordinal number of each object corresponds to the number from Table 1. $S$ is the diameter. The distances $D$ were taken from [1]. The spectral types in columns (6)-(9) correspond to [2], and those in columns (10) and (11) to [3]

\begin{tabular}{|c|c|c|c|c|c|c|c|c|c|c|}
\hline No. & $S, \mathrm{pc}$ & $D, \mathrm{kpc}$ & $n_{\mathrm{e}}, \mathrm{cm}^{-3}$ & $\begin{array}{c}\log Q_{\mathrm{Ly}}, \mathrm{s}^{-1} \\
\left(\log Q_{\min }, \log Q_{\max }\right)\end{array}$ & $T_{\mathrm{eff}}^{\mathrm{V}}, \mathrm{K}$ & Sp.typ. ${ }^{\mathrm{V}}$ & $T_{\text {eff }}^{\mathrm{III}}, \mathrm{K}$ & Sp.typ. ${ }^{\text {III }}$ & $T_{\text {eff }}^{\mathrm{V}^{*}}, \mathrm{~K}$ & Sp.typ. ${ }^{*}$ \\
\hline 2 & $2.8 \pm 2.8$ & $4.3 \pm 1.0$ & 255.98 & $48.44(47.36,48.72)$ & 35049 & 9.5 & 30999 & 0.5 & 70 & O8/O9 \\
\hline 4 & $1 \pm 2.5$ & $5.3 \pm 0.5$ & 127.23 & $48.73(47.66,49.01)$ & 37287 & O8.5/O9 & 32656 & O9.5/B0 & 37480 & O7/O7.5 \\
\hline 7 & $0 \pm 2.4$ & $3.2 \pm 0.6$ & 6797.51 & $48.56(47.52,48.84)$ & 35932 & O9/09.5 & 31628 & O9.5/B0 & 35380 & 08 \\
\hline 9 & $1.3 \pm 0.6$ & $12.9 \pm 0.5$ & 14.85 & $48.39(47.18,48.68)$ & 34678 & O9.5/B0 & 30751 & B0/B0.5 & 34178 & O7.5/O8 \\
\hline 13 & $2.8 \pm 1.7$ & $3.7 \pm 0.6$ & 367.12 & $48.88(47.86,49.16)$ & 38595 & O8/O8.5 & 33696 & O9/09.5 & 38880 & O7/O7.5 \\
\hline 34 & $1.2 \pm 0.6$ & $4.6 \pm 0.5$ & 474.90 & $47.98(46.84,48.27)$ & 32943 & B0/B0.5 & - & - & 32607 & O8/O9 \\
\hline 25 & $3.6 \pm 2.9$ & $15 \pm 0.8$ & 219.12 & $48.73(47.59,49.01)$ & 37270 & O8.5/O9 & 32643 & O9.5/B0 & 37480 & O7/O7.5 \\
\hline 27 & $4.3 \pm 2.6$ & $14.2 \pm 0.6$ & 186.18 & $48.77(47.63,49.05)$ & 37570 & O8.5/O9 & 32871 & O9.5/B0 & 37853 & O7/O7.5 \\
\hline 28 & $3.9 \pm 1.6$ & $12.2 \pm 0.4$ & 123.24 & $48.27(47.09,48.56)$ & 33977 & O9.5/B0 & 30160 & B0.5 & 33718 & O7.5/O8 \\
\hline 29 & $2.2 \pm 1.1$ & $13.4 \pm 0.5$ & 283.27 & $48.37(47.22,48.66)$ & 34548 & O9.5/B0 & 30641 & B0/B0.5 & 34102 & $\mathrm{O} 7.5 / \mathrm{O} 8$ \\
\hline 30 & $1.3 \pm 0.5$ & $4.5 \pm 0.4$ & 101.23 & $47.28(46.07,47.57)$ & - & - & - & - & 29570 & B0/B0.5 \\
\hline 35 & $1.9 \pm 0.8$ & $10.7 \pm 0.4$ & 201.72 & $47.79(46.55,48.08)$ & - & - & - & - & 31838 & O9.5/B0 \\
\hline 38 & $1.3 \pm 0.5$ & $10.4 \pm 0.4$ & 406.21 & $48.84(47.74,49.12)$ & 38191 & O7.5/O8 & - & - & 38507 & O7.5/O8 \\
\hline 48 & $2.0 \pm 1.0$ & $9.5 \pm 0.5$ & 333.97 & $48.24(47.08,48.53)$ & 31657 & B0/B0.5 & 29676 & O9.5/B0 & 33603 & O7.5/O8 \\
\hline 51 & $3.1 \pm 1.9$ & $9.2 \pm 0.6$ & 236.20 & $48.56(47.42,48.84)$ & 35882 & O9/09.5 & 31585 & O9.5/B0 & 35380 & O7.5/O8 \\
\hline 54 & $1.3 \pm 0.7$ & $9.7 \pm 0.5$ & 291.90 & $47.66(46.40,47.95)$ & - & - & - & - & 31292 & O9.5/B0 \\
\hline
\end{tabular}

*E-mail: ATopchieva@inasan.ru

**E-mail: kirsanova@inasan.ru

***E-mail: Andrej.Sobolev@urfu.ru

The original article can be found online at https://doi.org/10.1134/S1063772918110082 
Table 2. (Contd.)

\begin{tabular}{|c|c|c|c|c|c|c|c|c|c|c|}
\hline No. & $S, \mathrm{pc}$ & $D, \mathrm{kpc}$ & $n_{\mathrm{e}}, \mathrm{cm}^{-3}$ & $\begin{array}{c}\log Q_{\mathrm{Ly}}, \mathrm{s}^{-1} \\
\left(\log Q_{\min }, \log Q_{\text {max }}\right)\end{array}$ & $T_{\mathrm{eff}}^{\mathrm{V}}, \mathrm{K}$ & Sp.typ. ${ }^{\mathrm{V}}$ & $T_{\text {eff }}^{\mathrm{III}}, \mathrm{K}$ & Sp.typ. ${ }^{\text {III }}$ & $T_{\text {eff }}^{\mathrm{V}^{*}}, \mathrm{~K}$ & Sp.typ. ${ }^{*}$ \\
\hline 56 & $0.5 \pm 0.2$ & $4 \pm 0.4$ & 708.97 & $46.95(45.72,47.24)$ & - & - & - & - & 27920 & $\mathrm{~B} 0.5 / \mathrm{B} 1$ \\
\hline 57 & $3 \pm 1.5$ & $10.3 \pm 0.4$ & 199.74 & $48.54(47.41,48.82)$ & 35740 & O9/09.5 & 31464 & O9.5/B0 & 35120 & $\mathrm{O} 7.5 / \mathrm{O} 8$ \\
\hline 59 & $4.6 \pm 2.3$ & $5.5 \pm 0.5$ & 184.85 & $48.76(47.72,49.04)$ & 37539 & O8/O8.5 & 32828 & $\mathrm{O} 9.5 / \mathrm{B} 0$ & 37760 & O7.5/O8 \\
\hline 62 & $6 \pm 1.0$ & $8.9 \pm 0.6$ & 597.45 & $48.62(47.49,48.90)$ & 36351 & O8.5/O9 & 31944 & O9.5/B0 & 36160 & O7/O7.5 \\
\hline 64 & $4 \pm 1.3$ & $7.3 \pm 0.9$ & 363.17 & $47.94(46.75,48.23)$ & 33128 & $\mathrm{~B} 0 / \mathrm{B} 0.5$ & - & - & 32453 & O9 \\
\hline 65 & $1.0 \pm 0.9$ & $6.2 \pm 0.9$ & 786.50 & $48.10(46.92,48.39)$ & 32336 & B0/B0.5 & - & - & 33067 & O8/O9 \\
\hline 65 & $1.0 \pm 0.9$ & $6.2 \pm 0.9$ & 786.50 & $48.10(46.92,48.39)$ & 32336 & $\mathrm{~B} 0 / \mathrm{B} 0.5$ & - & - & 067 & O9 \\
\hline 68 & $5.4 \pm 4.3$ & $8.5 \pm 0.8$ & 57 & $48.38(47.24,48.66)$ & 34634 & B0 & 30714 & B0/B0.5 & 140 & O9 \\
\hline 69 & $4.2 \pm 2.5$ & $13.2 \pm 0.6$ & 73.67 & $48.00(46.73,48.29)$ & 32834 & B0/B0.5 & - & - & 32683 & O8/O9 \\
\hline 71 & $3.4 \pm 2.7$ & $10.8 \pm 0.8$ & 145.42 & $48.28(47.10,48.57)$ & 34021 & O9.5/B0 & 30195 & $\mathrm{~B} 0 / \mathrm{B} 0.5$ & 33757 & O9 \\
\hline 72 & $7 \pm 2.4$ & $.8 \pm 0.5$ & 81 & $48.59(47.42,48.88)$ & 29 & 9.5 & 31795 & O9.5/B0 & 770 & O8 \\
\hline 73 & $1.2 \pm 0.5$ & $10.6 \pm 0.4$ & 607.34 & $48.09(46.89,48.38)$ & 32416 & B0/B0.5 & - & - & 33028 & O8/O9 \\
\hline 74 & $5.1 \pm 2.6$ & $10.1 \pm 0.5$ & 102.72 & $48.52(47.40,48.80)$ & 35615 & O9/09.5 & 31358 & O9.5/B0 & 34860 & $\mathrm{O} 7.5 / \mathrm{O} 8$ \\
\hline 75 & $2.7 \pm 1.3$ & $11.0 \pm 0.5$ & & 47.90 & 50 & 5 & - & - & 300 & 9 \\
\hline 76 & $2.7 \pm 1.4$ & $10.8 \pm 0.5$ & 266.81 & $48.49(47.36,48.77)$ & 35388 & O9/O9.5 & 31234 & B0/B0.5 & 34562 & O8 \\
\hline 78 & $3.4 \pm 1.7$ & $10.1 \pm 0.5$ & 348.55 & $48.68(47.62,48.96)$ & 36865 & O8.5/O9 & 32335 & O9.5/B0 & 36940 & O8/O9 \\
\hline 79 & $1.6 \pm 0.9$ & $11.5 \pm 0.6$ & 676.56 & $48.38(47.20,48.67)$ & 34623 & O9.5/B0 & 30704 & $\mathrm{~B} 0 / \mathrm{B} 0.5$ & 34140 & O8/O9 \\
\hline 80 & $6.2 \pm 3.1$ & $9.2 \pm 0.5$ & 59.35 & $48.20(47.03,48.49)$ & 33574 & $\mathrm{O} 9.5 / \mathrm{B} 0$ & - & - & 33450 & $\mathrm{O} 8$ \\
\hline 82 & $0.4 \pm 0.2$ & $4.2 \pm 0.6$ & 895.04 & $47.13(45.89,47.42)$ & - & - & - & - & 28783 & B0/B0.5 \\
\hline 83 & $1.0 \pm 0.7$ & $1.3 \pm 0.7$ & 263.36 & $47.13(46.11,47.41)$ & - & - & - & - & 28783 & B0/B0.5 \\
\hline 85 & $1.4 \pm 7.2$ & $1.3 \pm 0.6$ & 79.53 & $48.75(47.61,49.04)$ & 37442 & O8/O8.5 & 32760 & $9.5 / \mathrm{B} 0$ & 37667 & O7.5/O8 \\
\hline 86 & $4.4 \pm 0.4$ & $6.1 \pm 0.1$ & 52.19 & $47.65(46.49,47.94)$ & - & - & - & - & 31250 & O9.5/B0 \\
\hline 87 & $5.9 \pm 0.6$ & $6.1 \pm 0.1$ & 53.62 & $48.27(47.17,48.55)$ & 33968 & O9.5/B0 & 30160 & B0.5 & 33718 & O8/O9 \\
\hline 88 & $1.0 \pm 2.2$ & $6.0 \pm 2.2$ & 537.61 & $47.69(46.54,47.98)$ & - & - & - & - & 31418 & O9.5/B0 \\
\hline 89 & $3.9 \pm 4.3$ & $16.2 \pm 1.1$ & 157.46 & $48.62(47.44,48.91)$ & 36381 & O8.5/O9 & 31967 & $\mathrm{O} 9.5 / \mathrm{B} 0$ & 36160 & O8/O9 \\
\hline 90 & $5.5 \pm 5.5$ & $5.8 \pm 1.0$ & 52.62 & $48.05(46.93,48.33)$ & 32580 & B0/B0.5 & - & - & 32875 & O8/O9 \\
\hline 91 & $3.5 \pm 2.4$ & $10.2 \pm 0.7$ & 146.99 & $48.36(47.20,48.65)$ & 34484 & O9.5/B0 & 30587 & $\mathrm{~B} 0 / \mathrm{B} 0.5$ & 34063 & O8/O9 \\
\hline
\end{tabular}

\section{REFERENCES}

1. L. D. Anderson, T. M. Bania, D. S. Balser, V. Cunningham, T. V. Wenger, B. M. Johnstone, and W. P. Armentrout, Astrophys. J. Supp. 212, id. 1 (2014).

2. W. D. Vacca, C. D. Garmany, and J. M. Shull, Astro- phys. J. 460, 914 (1996).

3. L. J. Smith, R. P. F. Norris, and P. A. Crowther, Monthly Not. Roy. Astron. Soc. 337, 1309 (2002).

Translated by D. Gabuzda 